\title{
How to develop a program to increase influenza vaccine uptake among workers in health care settings?
}

\author{
Ingrid Looijmans-van den Akker ${ }^{1}$, Marlies E Hulscher ${ }^{2}$, Theo JM Verheij ${ }^{1}$, Josien Riphagen-Dalhuisen², \\ Johan JM van Delden ${ }^{1}$ and Eelko Hak ${ }^{3^{*}}$
}

\begin{abstract}
Background: Apart from direct protection and reduced productivity loss during epidemics, the main reason to immunize healthcare workers (HCWs) against influenza is to provide indirect protection of frail patients through reduced transmission in healthcare settings. Because the vaccine uptake among HCWs remains far below the health objectives, systematic programs are needed to take full advantage of such vaccination. In an earlier report, we showed a mean $9 \%$ increase of vaccine uptake among HCWs in nursing homes that implemented a systematic program compared with control homes, with higher rates in those homes that implemented more program elements. Here, we report in detail the process of the development of the implementation program to enable researchers and practitioners to develop intervention programs tailored to their setting.

Methods: We applied the intervention mapping (IM) method to develop a theory- and evidence-based intervention program to change vaccination behaviour among HCWs in nursing homes.

Results: After a comprehensive needs assessment, we were able to specify proximal program objectives and selected methods and strategies for inducing behavioural change. By consensus, we decided on planning of three main program components, i.e., an outreach visit to all nursing homes, plenary information meetings, and the appointment of a program coordinator - preferably a physician - in each home. Finally, we planned program adoption, implementation, and evaluation.

Conclusion: The IM methodology resulted in a systematic, comprehensive, and transparent procedure of program development. A potentially effective intervention program to change influenza vaccination behaviour among HCWs was developed, and its impact was assessed in a clustered randomised controlled trial.
\end{abstract}

\section{Introduction}

Following 2004 guidelines by the World Health Organization, the Dutch association of nursing home physicians (Verenso) has been recommending influenza vaccination of healthcare workers (HCWs) [1]. In nursing homes, higher uptake of influenza vaccines has been associated with reduced morbidity and mortality among their frail patient population [2]. In a recent Cochrane review, an overall reduction in all-cause mortality of $32 \%$ ( $95 \%$ confidence interval 16 to $45 \%$ ) was

\footnotetext{
* Correspondence: e.hak@rug.nl

${ }^{3}$ University of Groningen, Department of Pharmacy, Pharmacoepidemiology and Pharmacoeconomy, A. Deusinglaan 1, 9713 AV, Groningen, The Netherlands

Full list of author information is available at the end of the article
}

found in long-term care facilities in which part of the HCWs were vaccinated versus control homes. One of the studies from that review [3] revealed that in the control homes in a sample of 30 deaths $20 \%$ was caused by influenza. In the intervention homes none of the sampled deaths had evidence of influenza infection, which corresponds with a $100 \%$ reduction in deaths caused by influenza. In addition, Thomas et al. obtained an estimate of $29 \%$ reduction (95\% confidence interval between 10 and 45\%) in influenza-like illness in intervention homes as compared with control homes. It has been well established that during influenza epidemics, the etiological fraction of culture or PCR-confirmed influenza virus in elderly patients is high - between $55 \%$

\section{Ciomed Central}


and $67 \%$ [4]. While immunisation of HCWs reduces the occurrence of influenza infections and associated productivity loss among the HCWs, it also ensures continuity of care during influenza epidemics [5-7]. A significant number of $\mathrm{HCW}$ s are infected with influenza each winter $[8,9]$, and most of them continue to work despite of infection $[5,10,11]$. Therefore, HCWs can introduce influenza in healthcare settings and increase the risk of an influenza outbreak. Such an outbreak in turn can have significant consequences for patients and continuity of care in healthcare institutions such as those with long-term care, including nursing homes [9]. In The Netherlands, HCWs with risk-elevating conditions are routinely invited by their primary care physician. However, the majority of HCWs (approximately 85 to $90 \%$ ) are otherwise healthy and despite recommendations to immunize this specific target group against influenza, vaccine uptake among HCWs in this high-risk setting remains far below the health objectives of $50 \%$ or more with estimated average vaccine uptake rates of $10 \%$ in 2005 [12].

To be most effective, implementation programs to change behaviour should be built upon a coherent theoretical base and should target all relevant determinants of influenza vaccine uptake among HCWs [13-15]. Previous programs targeting $\mathrm{HCW}$ s have, to our knowledge, not incorporated such a systematic approach. Often it remains unclear why specific interventions are chosen in implementation studies reported in the literature [16]. There are several more or less systematic methods available to develop implementation programs, including both exploratory methods (mainly based on brainstorming and consensus) and theory-based methods. One of these methods is the intervention mapping (IM) method, which offers a structured approach to develop theory- and evidence-based programs [17-19]. We used this IM method to systematically develop an intervention program to change vaccination behaviour among HCWs that could be implemented in nursing homes in the Netherlands. In an earlier report, we showed a mean $9 \%$ increase of vaccine uptake among HCWs in nursing homes that implemented the systematically developed program compared with control homes, with higher rates in those homes that implemented more program elements [20]. Here, we report in detail the process of the development of the implementation program to enable researchers and practitioners to develop intervention programs tailored to their setting.

\section{Methods}

The IM method is a framework developed in the field of health education and promotion to systematically design theory- and evidence-based health promotion programs
[17]. It was originally developed for interventions aimed at high-risk behaviours (e.g., HIV prevention [18]), and has also been used for other types of interventions (e.g., quality improvement interventions [19]). The IM method follows several consecutive steps giving planners a systematic method for decision making in each phase of intervention development [17]. The process of intervention design can be divided into six steps: a needs assessment; specification of proximal program objectives; selection of theory-based methods and practical strategies for inducing change; planning the program; planning of program adoption and implementation; and planning for evaluation. The steps of the IM method and their components are shown in Figure 1.

\section{Developing the program}

Step 1: needs assessment

To improve influenza vaccine uptake among healthcare workers (HCWs) of nursing homes, we first identified relevant barriers to and facilitators of vaccination uptake. These determinants may be related to the individual $\mathrm{HCW}$ or to the social, organisational, and economic context [16]. To explore all these levels, we organised three individual in-depth interviews with nursing home physicians and two focus group sessions (one with four nursing home physicians and one with three nursing assistants and two nurses). These were used to explore in a structured manner what determinants of influenza vaccination behaviour the participants experienced in daily practise. The structure of the sessions was based on both the theory of planned behaviour and the health behavioural model. Next, to complement these determinants identified by exploratory methods with theory-based determinants, we conducted an informal review of the international literature on determinants of influenza vaccine uptake among HCWs. Based on these qualitative methods, we conducted two quantitative questionnaire studies to specifically assess these determinants of vaccine uptake among HCWs in Dutch nursing homes.

\section{Questionnaire study one: Determinants of influenza vaccine uptake at management level}

The first questionnaire study was conducted among the management of all 335 nursing homes in the Netherlands with an average size of 178 patients and 232 HCWs assessing organisational determinants at management level known from the literature to be associated with higher influenza vaccine uptake among their HCWs [12]. The response rate was $45 \%$. In October 2005, the following items were assessed: uptake of influenza vaccination among patients and HCWs in the preceding season (2004 to 2005 season), whether the institution had a written policy on influenza vaccination for HCWs, what the current offering policy was (active 


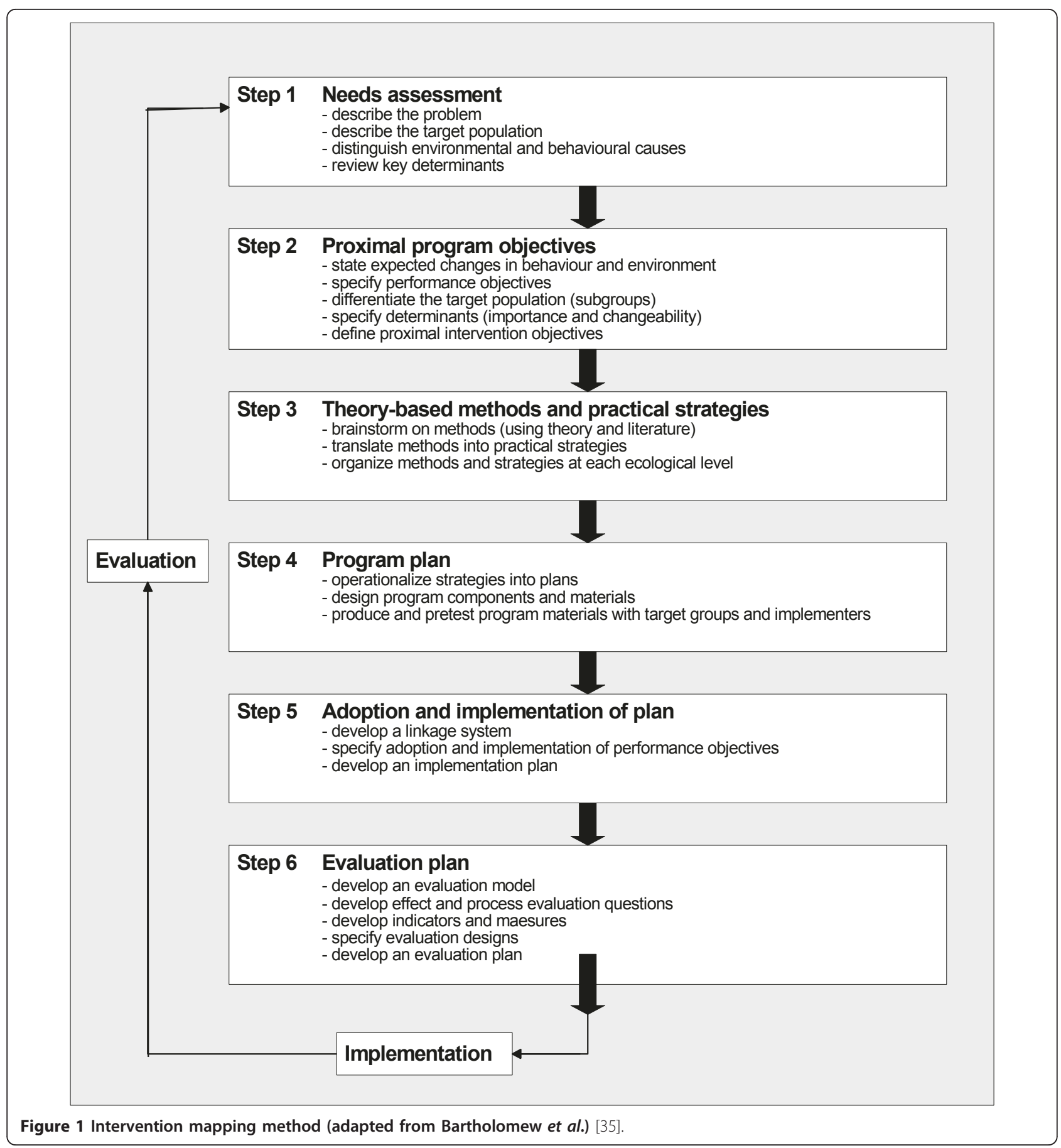

request, employees initiative, or none), and if HCWs were currently offered information on influenza vaccination.

\section{Questionnaire study two: Determinants of influenza vaccine uptake at $\mathrm{HCW}$ level}

The second study was a questionnaire study among HCWs of Dutch nursing homes assessing demographical, behavioural, and organisational determinants associated with uptake of influenza vaccination among HCWs. This questionnaire was based on the in-depth interviews and the focus group sessions, a review of the literature [21-28], and two previously developed questionnaires by our research group $[29,30]$. The questionnaire contained 12 questions on demographic determinants and 39 questions on behavioural determinants and on actual uptake of the vaccine. Questions on behavioural determinants were based on the 'Health Belief model' [31] and the 
'Behavioural Intention Model' [32]. These models were selected because results of the in-depth interviews and focus group sessions indicated that most participants experienced determinants on this individual level. The following five Health Belief Model domains were assessed: perceived susceptibility, perceived severity, perceived benefits, perceived barriers, and cues to action. These were complemented with the two Behavioural Intention Model domains: attitude (including ethical views) and social influences. Finally, six questions assessed organisational determinants consisting of the current situation concerning organisation of information on influenza vaccination (information received or not, route of information, and whether information has been sufficient or not), opinion towards various routes of receiving information, and the current situation concerning the organisation of vaccine provision (if and how provision is organised and if this has been adequate). In December 2005, these determinants of influenza vaccination uptake were assessed by an anonymous, self-administered, 59-item questionnaire. In all, 1,125 of 1,889 questionnaires were returned from the 32 randomly selected study nursing homes. The mean age and gender distribution of study participants was similar to total HCW personnel in The Netherlands, but less educated staff appeared underrepresented, but we could take this determinant into account in the prediction model.

These two combined studies resulted in a total of 73 possible determinants of influenza vaccine uptake relevant for the development of the intervention program: 70 determinants on HCW level (12 demographical, 39 behavioural, and 19 organisational determinants) and three organisational determinants on management level.

\section{Step two: Specification of proximal program objectives}

To specify our intervention objectives, we analysed the relation between all 73 possible determinants (step 0) and actual vaccine uptake. The outcomes of our first study on management level with a response rate of $45 \%$ (149 out of 335 nursing homes) showed that having a written policy, actively requesting HCWs to get vaccinated, and informing HCWs about influenza vaccination were all associated with a significantly higher uptake of influenza vaccination among HCWs [12]. Mean differences (MD) of these three determinants were reported as measures of associations (see Table 1).

The outcomes of our second study on HCW level with data from 1,125 respondents (response rate 60\%) enabled us to accurately predict influenza vaccine uptake on a HCW level based on a multivariate prediction model with 13 determinants (area under the receiver operating curve [AUC] of 0.95). This model included two demographical determinants, nine behavioural determinants, and two organisational determinants in which odds ratios (OR) were reported as measures of associations (see Table 1). The presence of chronic illness is a requirement for routine recommendations to be vaccinated by primary healthcare physicians in The Netherlands.

To quantify the 'importance' of the determinants resulting from both studies, we used the measures of association of the determinant with influenza vaccine uptake, i.e., the mean differences and odds ratios. We prioritised the importance of the determinants based on the strength of these associations (Table 1).

Next, to specify 'changeability,' we judged the changeability of the determinants based on consensus among all project group members (Table 1). Because the two demographical determinants (presence of chronic illness and working in healthcare for more than 15 years) were positively associated with influenza vaccine uptake, but not changeable, we did not define intervention objectives for these specific determinants nor used these determinants to define specific target subgroups.

Finally, the combination of importance and changeability of determinants was used to define intervention objectives (Table 2). For example, the determinant 'perceived high personal risk' was considered important (OR $=2.80$ ) and changeable and therefore 'accomplishing awareness among HCWs of being at risk for an influenza infection and knowledge on the height of this risk' was defined as an intervention objective.

\section{Step three: Selection of methods and strategies}

For the selection of theoretical methods and strategies we used the list of known types of implementation interventions from the EPOC data collection checklist [33]. In addition, we used both the general literature on the effectiveness of these different interventions [14] and the literature on previous studies that specifically tested intervention methods to increase influenza vaccine uptake among HCWs. A systematic review from 2006 evaluating whether promotional campaigns could improve uptake of influenza vaccination in HCWs was among the literature used [7]. Reviewing all available information, the project group finally decided on methods and strategies to be used in order to reach the intervention objectives (Table 2). For example, gender and presence of illness are associated with uptake, but cannot be changed. However, these determinants might be of use to define specific subgroups for the intervention. Perceived risk and potential reduction by vaccination can be changed by effective educational methods that focus on increasing knowledge like information leaflets, websites, group presentations, and videos with role models [13-15]. Ethical issues like 'do no harm' need to be targeted with more intensive activities such as small group discussions and role models in management of the centres. Social influence also asks for a more comprehensive approach that includes discussions at 
Table 1 Determinants resulting from the needs assessment and their importance and changeability

\begin{tabular}{|c|c|c|c|}
\hline & & Importance ${ }^{1}$ & Changeability $^{2}$ \\
\hline \multicolumn{4}{|c|}{ Determinants of influenza uptake at management level } \\
\hline a & Having a written policy & 4.58 & + \\
\hline$b$ & Actively requesting $\mathrm{HCW}$ s to get vaccinated & 6.77 & + \\
\hline c & Informing HCWs about influenza vaccination & 8.27 & + \\
\hline \multicolumn{4}{|c|}{ Determinants of influenza uptake at HCW level } \\
\hline \multicolumn{4}{|c|}{ Demographical } \\
\hline$d$ & Presence of chronic illness & 8.50 & - \\
\hline e & Working in health care for more than 15 years & 2.32 & - \\
\hline \multicolumn{4}{|c|}{ Behavioural } \\
\hline$f$ & Perceived high personal risk & 2.80 & + \\
\hline g & Perceived reduction of personal risk & 2.56 & + \\
\hline h & Perceived reduction of risk to infect patients & 3.29 & + \\
\hline i & Awareness of the existence of a guideline & 1.86 & + \\
\hline j & Agreement with this existing guideline & 2.75 & + \\
\hline k & Social influence of people close to the HCWs & 5.33 & - \\
\hline । & Influence of media attention for avian influenza & 2.24 & + \\
\hline $\mathrm{m}$ & All HCWs should get vaccinated & 2.25 & + \\
\hline $\mathrm{n}$ & HCWs should get vaccinated because of their duty not to harm & 4.71 & + \\
\hline \multicolumn{4}{|c|}{ Organisational } \\
\hline o & Information received through an information meeting & 3.40 & + \\
\hline $\mathrm{p}$ & Information received from a nursing home physician & 2.11 & + \\
\hline
\end{tabular}

management level and discussion evoking items such as videos with role models [13-15]. Finally, logistics need to be worked out to reduce efforts to get the vaccine like introduction of mobile carts.

\section{Step four: Planning of the program}

Next, the methods described in Table 2 were operationalized into practical strategies and materials (Table 3 ). By consensus, the intervention program consisted of three main components. Component A included an outreach visit during which homes were to receive a stepby-step script of the program, all required materials, and background information on influenza vaccination of HCWs. The required materials consisted of announcements, a personal invitation letter, leaflets, posters, and the reference to the programs' website. Component $B$ consisted of the plenary information meetings with a plenary presentation, discussion in smaller groups, and a video with role models. These meetings were to be organized by specialised nurses guided by a protocol. And, finally, component $\mathrm{C}$ prescribed the appointment of preferably a physician as a local program coordinator to organize and promote influenza vaccination.

All required materials were developed by our study group. The information leaflets and posters were developed in collaboration with the design department of the University Medical Center Utrecht and the information leaflet was pre-tested by three nursing assistants. They evaluated clearness, meaningfulness, and usefulness of the leaflet and were asked if any information was missing. The PowerPoint presentation for the information meetings, the leaflets, the posters, the video, and the website were all designed in a uniform style according to regulations of the University Medical Center Utrecht. For development of the website, we were assisted by our data management section. The video was recorded in a nursing home in Utrecht by a professional cameraman from the design department of the University Medical Center Utrecht. In the video, a nursing home physician, a nurse, and a patient shared their experiences on influenza and influenza vaccination with the viewers. The announcements and the personal invitation letter for the meetings were developed with standardised texts, leaving room to change dates, locations, and names according to the individual situation of nursing homes.

\section{Step five: planning of program adoption and \\ implementation}

To assure program adoption, implementation, and sustainability, stakeholders were approached to give feedback on and to support the program. Representatives of the Dutch association of nursing home physicians (Verenso) and the association of nurses and nursing assistants $(V \& V N)$ were approached to judge the different elements of the program. The V\&VN is the sole society for all Dutch nurses and nursing assistants with 36,000 
Table 2 Selected intervention objectives, methods and strategies

\begin{tabular}{lll}
\hline Determinants & Objectives & Methods and strategies \\
\hline $\begin{array}{l}\text { Management level } \\
\text { Having a written policy }\end{array}$ & $\begin{array}{l}\text { Stimulating nursing homes to develop a written } \\
\text { policy on influenza vaccination of HCWs }\end{array}$ & $\begin{array}{l}\text { Informing management on effect of a written policy (outreach } \\
\text { visit, written information) } \\
\text { Executing the intervention program automatically leads to an } \\
\text { actively requesting HCWs to request } \\
\text { get vaccinated }\end{array}$ \\
$\begin{array}{l}\text { Informing HCWs about } \\
\text { influenza vaccination }\end{array}$ & Having HCWs informed on influenza vaccination & $\begin{array}{l}\text { Informing HCWs by plenary meetings, discussion in smaller } \\
\text { groups, invitation letter, leaflets, posters, video, website }\end{array}$ \\
\hline
\end{tabular}

\section{HCW level}

Presence of chronic illness

No objective set due to limited changeability

Working in health care for more than 15 years

Perceived high personal risk

No objective set due to limited changeability

Awareness among HCWs of being at risk for an influenza infection and knowing how high this risk is

- Provide risk information (plenary meeting, leaflets, website)

- Show a video with role-models

Perceived reduction of personal risk

HCWs being convinced that vaccination is effective in reducing the personal risk for an influenza infection

- Provide effectiveness information concerning reduction of personal risk (plenary meeting, leaflets, posters, website)

- Interactive information provision by discussion in smaller groups

- Show a video with role models

Perceived reduction of risk to infect patients

HCWs being convinced that vaccination is effective in reducing the risk to infect patients with influenza

- Providing effectiveness information concerning the reduction of infecting patients (leaflets, posters, website, plenary meeting)

- Interactive information provision by discussion in smaller groups

- Show a video with role-models

Awareness of the existence of HCWs being aware of existence of guideline

Mention the existence of the guideline in program materials (leaflets, website, information meeting)

a guideline

Agreement with this guideline HCWs understanding reasoning of guideline

- Explain guideline (leaflets, website, plenary meetings)

- Discuss the guideline in smaller groups

Social influence of people

close to the HCWs

Also informing people close to the HCWs

Send a personal invitation letter for the plenary meetings to the home address of all HCWs together with an information leaflet

Influence of media attention for avian influenza

All HCWs should get

vaccinated

HCWs understand what avian influenza
it relates to annual human influenza

- Explain avian influenza on website

HCWs understand the ethical aspects of influenza vaccination among HCWs

- Explain and discuss ethical aspects (leaflets, website)

- Show a video with role-models

- Discussion in smaller groups

HCWs should get vaccinated because of their duty not to harm

HCWs understand the ethical aspects of influenza vaccination among HCWs

- Explain and discuss ethical aspects (leaflets, website)

- Show a video with role-models

- Discussion in smaller groups

Information received through Conducting an information meeting

Execute an information meeting with plenary information on influenza and influenza vaccination and discussion in smaller groups

Information received from a Having preferably a physician a local program Nursing home physician signing invitation letters and shows his support during information meetings

members (approximately 10\% of all HCWs in The Netherlands). They were asked for feedback on usefulness of the program elements and if program elements could be improved. This feedback was used to fine-tune the program elements, mainly by adjustment of difficulty of the language used. We did not translate the written information into other languages because most nurses in nursing homes understand the Dutch language. Support to the program was given byVerenso, $\mathrm{V} \& \mathrm{VN}$, and two other relevant healthcare management associations (Sting and ActiZ) and visualised with their logo on program materials (e.g., the information leaflets). Furthermore, to support future implementation of the program - without assistance by our study group - a step-by-step script of the total program was developed. In addition, the plenary information meetings were held by specialised nurses of the local municipal health centre guided by a standardised protocol. In The Netherlands these municipal health centers have a supportive role in the prevention of infection prevention in general and specifically influenza. In this protocol, we also included a list of frequently asked questions and corresponding answers.

We planned to send all 335 Dutch nursing homes an invitation letter to participate in the program mid-2006. 
Table 3 Components of the implementation program targeting determinants from Table 1

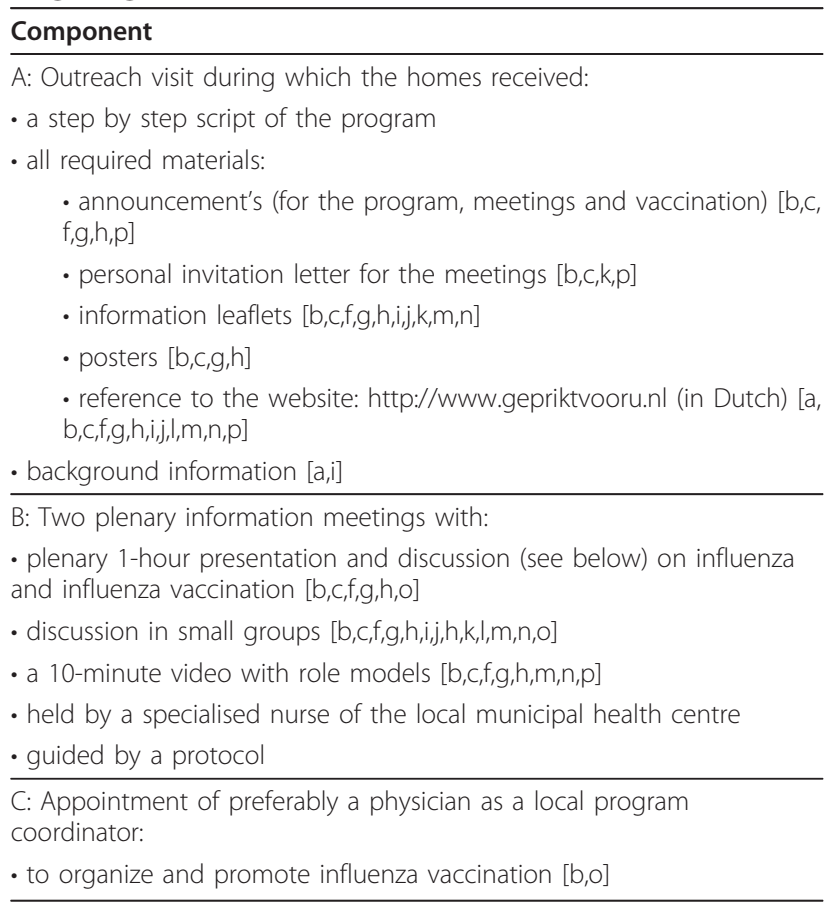

[ ]: determinants integrated in program component indicated by corresponding letter from Table 1.

Nursing homes who responded positive to this invitation $(\mathrm{n}=33$ ) would then be asked to appoint preferably a physician as local program coordinator (component C). Next, all nursing homes would be visited in September to deliver the step-by-step script of the program, all required materials, and the background information on influenza vaccination of HCWs (component A). Following these visits, execution of all program activities would be planned for October and November prior to the actual immunization of HCWs. During this period, the plenary information meetings were to be held by a specialised nurse of the local municipal health centre (component B).

\section{Step six: Planning for evaluation}

Our evaluation plan included both an effect and a process evaluation. We planned to evaluate the effectiveness of the program on influenza vaccine uptake among HCWs in Dutch nursing homes by comparing uptake in a group of at least 12 nursing homes randomly allocated to receive the intervention program with the uptake in a similar number of control homes. For this, we planned to perform a clustered randomised controlled trial [33]. In the trial, 33 nursing homes participated with a total of 6,636 HCWs. Mean number of patients per home were 160 and $200 \mathrm{HCW}$ s. Percentage of females was $90 \%$ and mean age was 40 years. The mean vaccine uptake in both intervention and control homes was $11 \%$ at baseline in 2005. In all, these figures were similar to The Netherlands as a whole. Furthermore, we decided to measure compliance with the programme components (process evaluation). For this purpose, we planned to register whether nursing homes were visited, whether plenary information meetings were organised, and how many HCWs visited these meetings, what the profession of the local program coordinator was, and, finally, all costs related to the program. In this manner we could, on the one hand, explore whether compliance with the components of the program influenced the effectiveness of the program. This information can be used to, if necessary, adapt the program. On the other hand, we could use this information to estimate program costs.

\section{Discussion}

This paper presents the process by which a theory- and evidence-based intervention was developed to improve influenza vaccination behaviour among HCWs. The IM method was used to systematically develop this intervention.

A major strength of the developing process was the comprehensive and thorough needs assessment that clearly identified relevant determinants for influenza vaccine uptake among $\mathrm{HCW}$ s on both management and $\mathrm{HCW}$ level. Combining explorative and theory-based methods assured that determinants not anticipated beforehand were included in the needs assessment and helped broaden the scope of this needs assessment. Based on the results of the needs assessment, we were able to quantify the importance of the determinants. This provided an anchor for the specification of program objectives and the successful further development of the intervention.

The selection of methods and strategies (step three of the IM method) is challenging, because specific objectives can ask for a variety of different interventions, and consensus is needed by the developers on the intervention with presumed highest impact [14]. As yet, there is no firm guidance on what interventions should be linked to what specific objectives. To facilitate this process, we considered the 'Health Belief model' [31] and the 'Behavioural Intention Model' [31], and the available evidence regarding the effectiveness of interventions $[14,33]$ combined with common sense and creativity to reach consensus.

Our systematic, comprehensive, and transparent description of all the steps in the development of the program enables future users to assess and adapt the program where necessary or to replicate the steps described when developing a similar program for a different population. Applying the systematic, comprehensive, and transparent IM approach guided and facilitated our development process. It may seem elaborate and 
time consuming, but a review of health promotion intervention studies has shown that the quality of planning is important for the success of the intervention [34,35]. Recently, we published our paper on the effects of the randomized controlled trial of this developed intervention program [20]. We showed that the intervention program resulted in a significantly higher $(25 \%$ in intervention group versus $16 \%$ in control group), though moderate, influenza vaccine uptake among HCWs in nursing homes.

\section{Acknowledgements and funding}

We great fully acknowledge the participation of the nursing home management and personnel in participating in this study.

The study was funded by the Netherlands Healthcare Research Organization ZONMW.

\section{Author details}

'Julius Center for Health Sciences and Primary Health Care, University Medical Center Utrecht, HP 6.131, POBOX 85500, 3508 GA Utrecht, The Netherlands. ${ }^{2}$ Scientific Institute for Quality of Healthcare (IQ Healthcare), Radboud University Nijmegen Medical Centre, POBOX 9101, 114 IQ Healthcare, 6500 HB Nijmegen, The Netherlands. ${ }^{3}$ University of Groningen, Department of Pharmacy, Pharmacoepidemiology and Pharmacoeconomy, A. Deusinglaan 1, 9713 AV, Groningen, The Netherlands.

\section{Authors' contributions}

ILVdA conducted the questionnaire studies and wrote the paper. MEH supervised the design of the program and drafts of the paper. TJMV commented on the paper. JRD critically commented on the paper and revised earlier drafts. JJVD and EH contributed both equally to the design of the program, were involved in earlier drafts, supervised the project and final draft. All authors have read and approved the final manuscript.

\section{Competing interests}

The authors declare that they have no competing interests.

Received: 18 October 2010 Accepted: 19 May 2011

Published: 19 May 2011

\section{References}

1. Cools HJ, van Essen GA: Practice guideline 'Influenza prevention in nursing homes and care homes,' issued by the Dutch Society of Nursing Home Specialists [article in Dutch, Englisch abstract]. Ned Tijdschr Geneeskd 2005, 149:119-124.

2. Thomas RE, Jefferson T, Lasserson TJ: Influenza vaccination for healthcare workers who work with the elderly. Cochrane.Database.Syst.Rev 2010, 2 CD005187.

3. Carman WF, Elder AG, Wallace LA, McAulay K, Walker A, Murray GD, Stott DJ: Effects of influenza vaccination of health-care workers on mortality of elderly people in long-term care: a randomised controlled trial. Lancet 2000, 355:93-97.

4. Call SA, Vollenweider MA, Hornung CA, Simel DL, McKinney WP: Does this patient have influenza? JAMA 2005, 293:987-997.

5. Van den Dool C, Bonten MJ, Hak E, Heijne JC, Wallinga J: The effects of influenza vaccination of health care workers in nursing homes: insights from a mathematical model. PLoS Med 2008, 5:e200.

6. Wilde JA, McMillan JA, Serwint J, McMillan JA, Serwint J, Butta J, O'Riordan MA, Steinhoff MC: Effectiveness of influenza vaccine in health care professionals: a randomized trial. JAMA 1999, 281:908-913.

7. Saxen H, Virtanen M: Randomized, placebo-controlled double blind study on the efficacy of influenza immunization on absenteeism of health care workers. Pediatr Infect Dis J 1999, 18:779-783.

8. Burls A, Jordan R, Barton P, Olowokure B, Wake B, Albon E, Hawker J: Vaccinating healthcare workers against influenza to protect the vulnerable-ls it a good use of healthcare resources? A systematic review of the evidence and an economic evaluation. Vaccine 2006, 24:4212-4221.
9. Bush KA, McAnulty J, McPhie K, Reynolds R, Boomer M, Clarkson LM, Quaine J, Dwyer DE: Southern New South Wales Public Health Unit. Antiviral prophylaxis in the management of an influenza outbreak in an aged care facility. Commun Dis Intell 2004, 28:396-400.

10. Mitchell R, Huynh V, Pak J, Thompson S, Noseworthy AL: Influenza outbreak in an Ontario long-term care home-January 2005. Can Commun Dis Rep 2006, 32:257-262.

11. Weingarten S, Staniloff H, Ault M, Miles P, Bamberger M, Meyer RD: Do hospital employees benefit from the influenza vaccine? A placebocontrolled clinical trial. J Gen Intern Med 1988, 3:32-37.

12. Christini $A B$, Shutt $K A$, Byers KE: Influenza vaccination rates and motivators among healthcare worker groups. Infect Control Hosp Epidemiol 2007, 28:171-177.

13. Looijmans-van den Akker I, van Delden JJ, Hak E: Uptake of influenza vaccination in Dutch nursing home personnel following national recommendations. J Am Geriatr Soc 2007, 55:1486-1487.

14. Grol R, Berwick DM, Wensing M: On the trail of quality and safety in health care. BMJ 2008, 336:74-76.

15. Grimshaw JM, Thomas RE, Maclennan G, Fraser C, Ramsay CR, Vale L, Whitty P, Eccles MP, Matowe L, Shirran L, Wensing M, Dijkstra R, Donaldson C: Effectiveness and efficiency of guideline dissemination and implementation strategies. Health Technol Assess 2004, 8:iii-iv, 1-72.

16. Grol R: Personal paper. Beliefs and evidence in changing clinical practice. BMJ 1997, 315:418-421.

17. Bartholomew LK, Parcel GS, Kok G: Intervention mapping: a process for developing theory- and evidence-based health education programs. Health Educ Behav 1998, 25:545-563.

18. Van Empelen P, Kok G, Schaalma HP, Bartholomew LK: An AIDS risk reduction program for Dutch drug users: an intervention mapping approach to planning. Health Promot Pract 2003, 4:402-412.

19. Van Bokhoven MA, Kok G, van der Weijden T: Designing a quality improvement intervention: a systematic approach. Qual Saf Health Care 2003, 12:215-220.

20. Looijmans-van den Akker I, van Delden JJ, Verheij TJ, van der Sande MA van Essen GA, Riphagen-Dalhuisen J, Hulscher ME, Hak E: Effects of a multi-faceted program to increase influenza vaccine uptake among health care workers in nursing homes: A cluster randomised controlled trial. Vaccine 2010, 28:5086-92.

21. Saluja I, Theakston KD, Kaczorowski J: Influenza vaccination rate among emergency department personnel: a survey of four teaching hospitals. CJEM 2005, 7:17-21.

22. Canning HS, Phillips J, Allsup S: Health care worker beliefs about influenza vaccine and reasons for non-vaccination-a cross-sectional survey. J Clin Nurs 2005, 14:922-925.

23. Mah MW, Hagen NA, Pauling-Shepard K, Hagen NA, Pauling-Shepard K, Hawthorne JS, Mysak M, Lye T, Louie TJ: Understanding influenza vaccination attitudes at a Canadian cancer center. Am J Infect Control 2005, 33:243-250.

24. O'Reilly FW, Cran GW, Stevens AB: Factors affecting influenza vaccine uptake among health care workers. Occup Med (Lond) 2005, 55:474-479.

25. Hofmann F, Ferracin C, Marsh G, Marsh G, Dumas R: Influenza vaccination of healthcare workers: a literature review of attitudes and beliefs. Infection 2006, 34:142-147.

26. Stephenson I, Roper JP, Nicholson KG: Healthcare workers and their attitudes to influenza vaccination. Commun Dis Public Health 2002, 5:247-252.

27. McEwen M, Farren E: Actions and beliefs related to hepatitis B and influenza immunization among registered nurses in Texas. Public Health Nurs 2005, 22:230-239.

28. Harrison J, Abbott P: Vaccination against influenza: UK health care workers not on-message. Occup Med (Lond) 2002, 52:277-279.

29. Hak E, Schonbeck Y, De Melker H, Van Essen GA, Sanders EA: Negative attitude of highly educated parents and health care workers towards future vaccinations in the Dutch childhood vaccination program. Vaccine 2005, 23:3103-3107.

30. Opstelten W, Hak E, Verheij TJ, Van Essen GA: Introducing a pneumococcal vaccine to an existing influenza immunization program: vaccination rates and predictors of noncompliance. Am J Med 2001, 111:474-479.

31. Rosenstock IM: The health belief model and preventive health behavior. In The health belief model and personal health behavior. Edited by: Becker MA. Thorofare: Charles B. Slack; 1974:27-59. 
32. Fishbein M, Azjen I: Belief, attitude, intention and behavior: an introduction to theory and research. Reading: Addison-Wesley; 1975.

33. Looijmans-van den Akker I, van Delden JJ, Verheij TJ, van der Sande MA, van Essen GA, Riphagen-Dalhuisen J, Hulscher ME, Hak : Effects of a multifaceted program to increase influenza vaccine uptake among health care workers in nursing homes: A cluster randomised controlled trial. Vaccine 2010, 28:5086-92.

34. Van Empelen P, Kok G, van Kesteren NM, van den Borne B, Bos AE, Schaalma HP: Effective methods to change sex-risk among drug users: a review of psychosocial interventions. Soc Sci Med 2003, 57:1593-1608.

35. Bartholomew LK, Parcel GS, Kok G, Gottlieb N: Intervention Mapping: A process for designing theory- and evidence-based health education programs. Mountain View, CA: Mayfield; 2001.

doi:10.1186/1748-5908-6-47

Cite this article as: Looijmans-van den Akker et al:: How to develop a program to increase influenza vaccine uptake among workers in health care settings? Implementation Science 2011 6:47.

\section{Submit your next manuscript to BioMed Central} and take full advantage of:

- Convenient online submission

- Thorough peer review

- No space constraints or color figure charges

- Immediate publication on acceptance

- Inclusion in PubMed, CAS, Scopus and Google Scholar

- Research which is freely available for redistribution

Submit your manuscript at www.biomedcentral.com/submit 